\title{
Gene therapy needs a long-term approach
}

\author{
Gene-therapy trials are on the rise, but more needs to be done to understand the long-term risks associated with \\ this type of treatment.
}

O n 16 February 2021, Bluebird Bio suspended phase $1 / 2$ and phase 3 clinical trials of its LentiGlobin gene therapy for sickle-cell disease after two patients were diagnosed with cancer, 5 years after receiving treatment. Earlier, in late 2018, another participant from the same trial had been diagnosed with myelodysplastic syndrome. Although further review seems to indicate that the lentiviral vector used in the studies is unlikely to be the cause of cancer, this news highlights growing concerns about the late adverse effects associated with gene therapy. Clinical use of gene therapies is rapidly increasing, and it is estimated that by 2025 , the US Food and Drug Administration (FDA) will be approving between 10 and 20 gene therapies each year. This fast pace of approvals must be matched by the development of new clinical frameworks for adequately assessing and managing the potential delayed effects of these new therapies.

Safety concerns associated with gene therapy are not new and have been highly publicized. The tragic death of Jesse Gelsinger in late 1999, during an early safety trial of a gene therapy for ornithine transcarbamylase deficiency, led to the near abandonment of the approach. But Peter Marks, director of the Center for Biologics Evaluation and Research at the FDA, urges caution in drawing parallels to the events of 20 years ago. He points out that considerable advances have been made in the understanding of viral vectors, culminating in the recent approval of promising gene therapies for diverse indications such as neuromuscular disease, inherited blindness, and cancer. He also remarks that so far, given the relatively large amount of work in the field, few safety concerns have been reported for these therapies, and the results of these trials are life-changing for many patients suffering rare and debilitating diseases. Care should be taken not to draw premature conclusions about the safety of this emerging therapy, which may impede the substantial progress seen in the field so far. Nonetheless, although advances have been made in the management of acute effects related to gene therapies, the potential for delayed effects remains a concern.

Most gene therapies are designed to achieve permanent or long-lasting effects in the human body, and this inherently increases the risk of delayed adverse events. Delayed adverse events associated with gene therapy have been reported before, such as in clinical trials for X-linked severe combined immunodeficiency. Recent long-term studies of animals suggesting that insertional mutagenesis could take years to be become apparent have raised more red flags. For full understanding and mitigation of the risk of a delayed adverse event, participants in gene therapy trials will need to be monitored for an extended period of time-commonly referred to as the 'long-term follow-up' period of a clinical study.

In 2020 the FDA updated guidelines on the design of long-term follow-up studies for the collection of data on delayed adverse events following the administration of a gene therapy product. The new guidelines suggest that studies using integrating vectors and genome-editing products follow patients for at least 15 years, while for adeno-associated viral vectors, a minimum 5-year follow-up period is recommended. All follow-up studies should have a dedicated clinical protocol that specifies patient-visitation schedules, a sampling plan, the methodology that will be used to assess the persistence of vector sequences, the clinical events that will be monitored, and a means for collecting accurate case histories. These recommendations are aligned with those suggested by European Union regulators, whereby gene-therapy trials also must adhere to updated guidelines on good clinical practice specific to advanced therapy medicinal products.

The guidelines from both US and EU regulatory bodies are excellent for establishing follow-up time frames and protocols. But these long-term follow studies must go beyond being an additional regulatory obstacle necessary for clinical approval of a product. It is important to take into account life expectancy based on the underlying disease and the possibility of multiple comorbidities or exposure to other agents that may have their own long-term adverse effects. Bluebird Bio's clinical studies, for example, required that patients be pre-conditioned with a chemotherapy before administration of the gene therapy. Monitoring the persistence of therapeutic outcomes is also important, as it is unclear if all therapies have the same persistence characteristics in humans.

The considerable time periods associated with long-term follow-up studies will require that researchers develop innovative follow-up strategies to keep patients engaged for up to 15 years. This may include the use of mobile health applications to improve the self-reporting of symptoms or fostering stronger relationships with foundations that already have strong links to patients.

For maximization of the impact of these studies, data need to be shared openly and rapidly with the scientific community. Sharing of these clinical data enhances the safety and efficacy of gene therapies moving forward by reducing the exposure of participants in future trials to avoidable harms and reducing unnecessary duplication of effort and the costs of future studies. This includes the sharing of negative data or findings from trials that may not have proceeded to approval. There are numerous examples of how sharing of secondary analyses generates better evidence on the safety and effectiveness of therapies for patients. Delayed or incomplete sharing of data is not only a safety risk for patients, it also prevents investigators from building on previous work and thereby slows progress in the understanding of human health.

Mila Makovec, the young girl who made headlines around the world for being the recipient of a custom-made gene therapy for her rare and fatal brain disease in 2018, passed away this year. Her story highlighted the advances made with gene therapies and paved the way for initiatives dedicated to making gene therapy a reality for those with rare genetic diseases. As is true with the development of any type of therapeutic, there will always be risks and challenges to overcome. But adopting a sound framework now to understand what happens with patients who undergo gene therapy will in the long run help to balance the risk and will provide valuable insight into how the long-term safety of gene-therapy products can be improved for the benefit of most patients.

Published online: 15 April 2021 https://doi.org/10.1038/s41591-021-01333-6 